\title{
The Effects of Surface Mechanical Deformation and Bovine Serum Albumin on the Tribological Properties of Polyvinyl Alcohol Hydrogel as an Artificial Cartilage
}

\author{
Feng Li, ${ }^{1}$ Gang Zhang, ${ }^{1}$ Anmin Wang, ${ }^{1}$ and Feng Guo ${ }^{2}$ \\ ${ }^{1}$ College of Electromechanical Engineering, Qingdao University of Science and Technology, Qingdao, Shandong 266061, China \\ ${ }^{2}$ School of Mechanical Engineering, Qingdao University of Technology, Qingdao, Shandong 266520, China \\ Correspondence should be addressed to Feng Guo; mefguo@qut.edu.cn
}

Received 23 June 2017; Revised 1 October 2017; Accepted 12 October 2017; Published 28 December 2017

Academic Editor: Hongchao Kou

Copyright (c) 2017 Feng Li et al. This is an open access article distributed under the Creative Commons Attribution License, which permits unrestricted use, distribution, and reproduction in any medium, provided the original work is properly cited.

\begin{abstract}
The mechanical and tribological properties of polyvinyl alcohol hydrogel as an artificial cartilage were studied under water and bovine serum albumin-lubricated sliding conditions. The frictional properties of the polyvinyl alcohol hydrogel were investigated via reciprocating frictional tests. The effect of surface mechanical deformation on the tribological properties of the polyvinyl alcohol hydrogel as an artificial cartilage was studied by concurrently recording the $z$-axis displacement and friction coefficient time. Three different factors were chosen including load, speed, and lubrication. The results showed that the albumin solution could reverse the trend in the coefficient of friction in tests at different loading levels. There was no improvement in the friction condition in albumin at low speeds. However, when the speed was increased to $2 \mathrm{~Hz}$, the coefficient of friction was significantly reduced. Wear testing was also conducted, and wear tracks were found on the sample surface. The results also showed that even though the surface deformation could recover as the water phase of the porous structure recovered, the coefficient of friction continued to increase simultaneously. This relationship between mechanical and frictional tests indicated that biphasic lubrication effects may not be the only dominant factor underlying the excellent friction properties of polyvinyl alcohol hydrogel.
\end{abstract}

\section{Introduction}

Many patients with osteoarthritis or advanced rheumatoid disease suffer from severe joint pain and immobility. Mobility and workplace efficiency will decrease when the injuries resulting from joint disease are severe. The most common therapy is to perform total joint arthroplasty or hemiarthroplasty that replaces the entire joint or parts of the natural joints with different artificial components. This treatment is a practical treatment for patients with severe joint disease. Unfortunately, the complications of joint replacement are always related to tribological failure [1]. Failures in joint replacement include pain and wear of the joint material including the natural articular cartilage and the artificial joint material [2-5]. Histological examination of the peri-implant tissue revealed some wear in the polyethylene particles. This is because the damaged articular cartilage of the joint surface has been surgically replaced by artificial cartilage, and the surface material of the joint has been worn out.

Articular cartilage covers the end of the joint surface and is a living material with a porous structure containing roughly $70 \%-85 \%$ of its weight in water [6]. Articular cartilage has a particularly complex structure and complicated chemical composition. The frictional properties of articular cartilage are of considerable interest to scientists and engineers in mechanical engineering or materials science for nearly a century. To reduce the severe wear phenomenon of the material in the artificial joint surface, some new types of artificial joint materials that could replace natural cartilage have recently been developed.

The synthetic articular cartilage from the polymer hydrogel was developed and studied [7]. Hydrogels are porous materials with a three-dimensional network structure. Hydrogels have similar properties as articular cartilage including compliance, hydrophilicity, and swelling capacity 


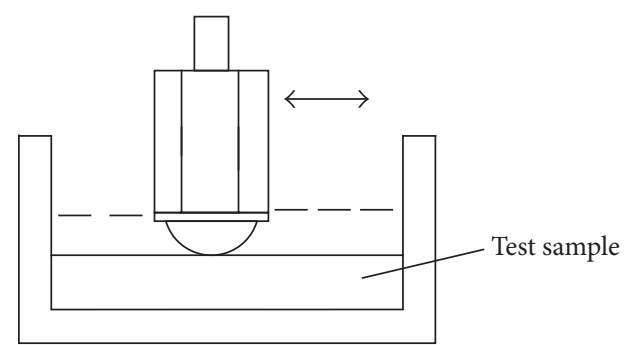

Figure 1: Schematic of friction tests.

TABLE 1: Factors and levels for the design of experiments.

\begin{tabular}{lccc}
\hline Factor & Level 1 & Level 2 & Level 3 \\
\hline Load $(\mathrm{N})$ & 1 & 2 & 3 \\
Speed $(\mathrm{Hz})$ & $1(10 \mathrm{~mm} / \mathrm{s})$ & $2(20 \mathrm{~mm} / \mathrm{s})$ & - \\
Lubricant & Deionized water & Albumin solution $(1 \mathrm{~g} / \mathrm{dl})$ & - \\
\hline
\end{tabular}

[8-10]. The fluid content in the solid matrix of articular cartilage is mainly water, and it helps the synovial joint to markedly reduce the friction and wear between cartilage counterparts.

The FDA (Food and Drug Administration, USA) has approved the biomedical application of polyvinyl alcohol (PVA) hydrogel in various instruments including medical membranes, drug delivery vehicles, and contact lenses $[11,12]$. There is a fairly similar structure pattern in the porous tissue of hydrogels [13], and broken cartilage might be able to be replaced by hydrogels in surgery [14-16]. Recently, the artificial meniscus has been developed via PVA hydrogels to replace damaged rabbit joints [17]. The results showed that the meniscus without any break was in good condition. This proved that the polyvinyl alcohol hydrogel could be used in synovial joints for medical purposes.

The aim of this study was to investigate the frictional and mechanical properties of PVA hydrogels as artificial cartilage when the synovial component was used as a lubricant. To achieve our research aims, experiments were performed to examine the tribological behavior between PVA hydrogels and stainless steel in an albumin solution and deionized water via a reciprocating motion. The coefficient of friction was recorded and evaluated in each test. In addition, the surface morphology and interior structure were tested by scanning electron microscopy.

\section{Materials and Methods}

2.1. Raw Materials. Polyvinyl alcohol (PVA) resin and dimethyl sulfoxide (DMSO) were purchased from Sinopharm Chemical Reagent Co., Ltd. (SCRC), China. The PVA had a viscosity average degree of polymerization of $1750 \pm 50$. The PVA had a saponification degree of $99 \%$. The PVA is a water-soluble and nontoxic polymer. It has excellent chemical stability and is widely used in medical products. A stainless steel ball $(316 ; 4 \mathrm{~mm}$ diameter) was used as the counterpart in the friction tests. Bovine serum albumin (BSA) was supplied by Beijing HMK Biotechnology Co., Ltd., China, with a molecular weight of $68 \mathrm{kDa}$.

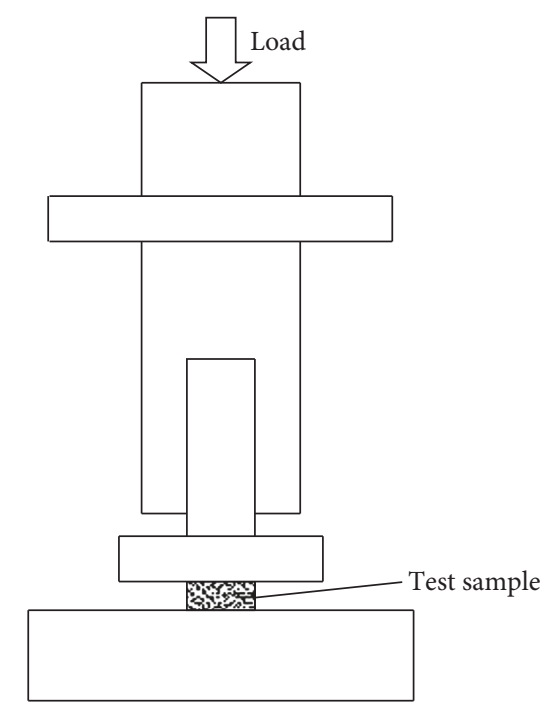

Figure 2: Schematic of compression tests.

2.2. Preparation of Specimen. PVA hydrogel was prepared via freezing and unfreezing cycles [18]. We used an aqueous mixture of PVA and dimethyl sulfoxide (DMSO) solution to obtain a PVA hydrogel via freezing. The ratio of DMSO and water was 80 wt. $\%$ and 20 wt. $\%$, respectively. The weight proportion of PVA was $15 \mathrm{wt} . \%$. The preparation of the PVA hydrogel includes several procedures. First, the mixture of PVA and DMSO solution was stirred at $100^{\circ} \mathrm{C}$ for more than 3 hours. Next, the solution was rapidly added to the stainless steel mold, which was put in a medical refrigerator for 24 hours at $-20^{\circ} \mathrm{C}$. Finally, the mold was warmed to room temperature, and the PVA hydrogel was removed from the mold and put into distilled water for more than 20 days to exchange the DMSO solvent. PVA hydrogel samples were stored in distilled water and immersed in a lubricant for 15 minutes before each test. The specimens were $20 \mathrm{~mm}$ long, $15 \mathrm{~mm}$ wide, and $3 \mathrm{~mm}$ thick.

2.3. Instrument and Methodology. The coefficient of friction between artificial articular cartilage and stainless steel ball was measured with a UMT-3 Micro-Tribometer (CETR Inc., USA) using a ball-plate reciprocating movement (Figure 1). The stainless steel ball was $4 \mathrm{~mm}$ and was placed on a fixture on the tribometer. The PVA hydrogel sample was stuck on a stainless steel plate that could be fixed on a reciprocating table dragged by a back-actuating motor. The reciprocating distance was $5 \mathrm{~mm}$. In the beginning of the experiment, the PVA hydrogel samples were prepared and submerged in the container filled with lubricant for more than ten minutes. The thickness of the specimen was measured with calipers. Three factors were chosen including load, speed, and lubrication. There were two levels for the lubricant and the speed. The load has three levels. Each test was conducted three times for 6 minutes. In addition, a long-term wear test of 60 minutes was conducted to examine the surface changes in the frictional surface.

In the reciprocating frictional tests, the displacement of the $z$-axis was also measured during each test. The reciprocating movement was used in the tester and was designed to 


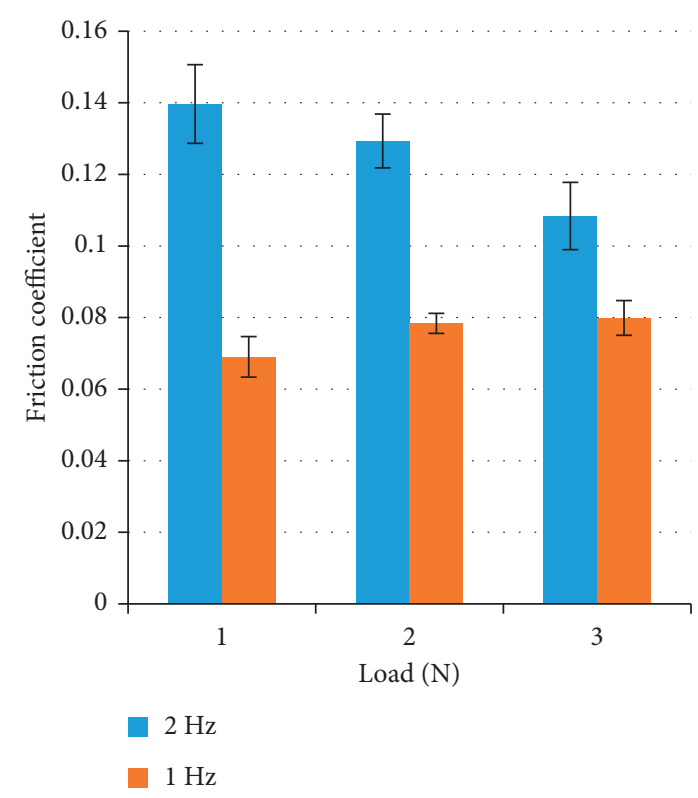

FIGURE 3: Effect of load under deionized water lubrication condition.

accurately simulate the joint activities in daily life. Although the mode of motion was simple, it accurately recapitulated the frictional performance of natural or artificial materials [19]. The frictional data and surface change were obtained through these experiments. The coefficient of friction was acquired from the whole reciprocating cycles of the tests. The value of the friction coefficient was calculated by $u=F / N$, where $u, N$, and $F$ are the friction coefficient, load, and friction force, respectively. The value of the coefficient of friction is the mean value.

The factors and levels for the designed experiments are shown in Table 1. In earlier studies, the range of natural normal joint contact stresses was about $0.1-2.0 \mathrm{MPa}[20,21]$. Katta et al. [22] used the contact stress ranging from 0.2 to $0.4 \mathrm{MPa}$ to evaluate the friction properties of the articular cartilage. Pan et al. [7] used a contact stress of $0.37-1.32 \mathrm{MPa}$ to study the frictional properties of the PVA hydrogel. Here, we used Hertzian contact theory and contact stresses of $0.4-0.6 \mathrm{MPa}$, which is the range of normal contact stress. The reciprocating sliding speed was from $10 \mathrm{~mm} / \mathrm{s}$ to $20 \mathrm{~mm} / \mathrm{s}$ to model human motions such as walking (normal knee joints) [23].

The lubricants used here were deionized water and albumin $(1 \mathrm{~g} / \mathrm{dl})$. Synovial fluid is an efficient lubricant that can reduce cartilage friction in natural joints. It is necessary to study the role of proteins in synovial fluids including albumin. Albumin had been studied in film thickness experiments, and its concentration is $0.7-1.8 \mathrm{~g} / \mathrm{dl}$ in healthy synovial fluid $[24,25]$.

The sample topography and worn surface in the friction tests were observed via scanning electron microscopy. The sample was freeze-dried to remove water and coated for scanning electron microscopy [26] (HITACHI S-3500N).

Surface roughness was measured by a TIME3200 profilometer (Time Group Inc., China) to evaluate the surface change in the tests. The sampling length used in the experiments was $0.8 \mathrm{~mm}$. This method could not break or destroy the sample surface. The results were reported as the average value in the measurements.

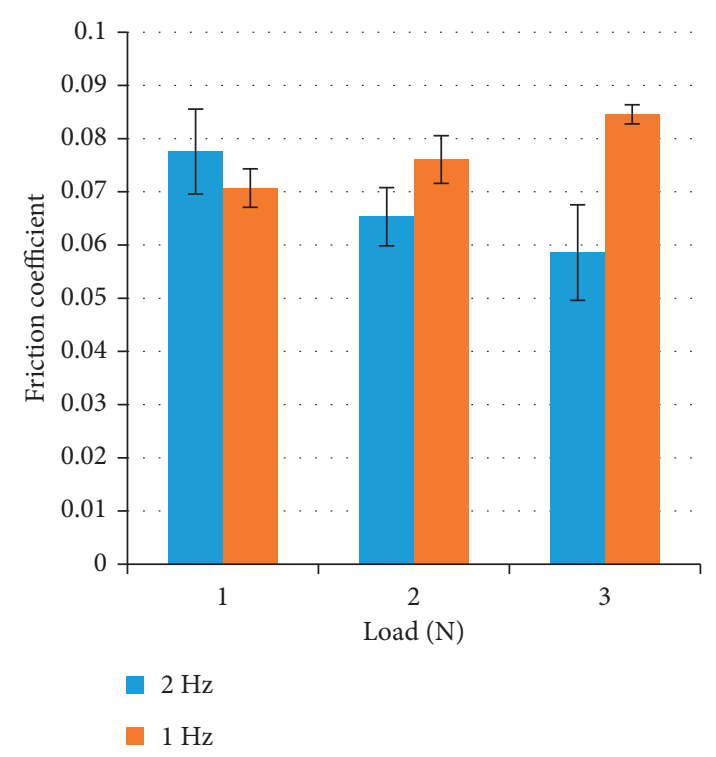

FIGURE 4: Effect of load under albumin solution lubrication.

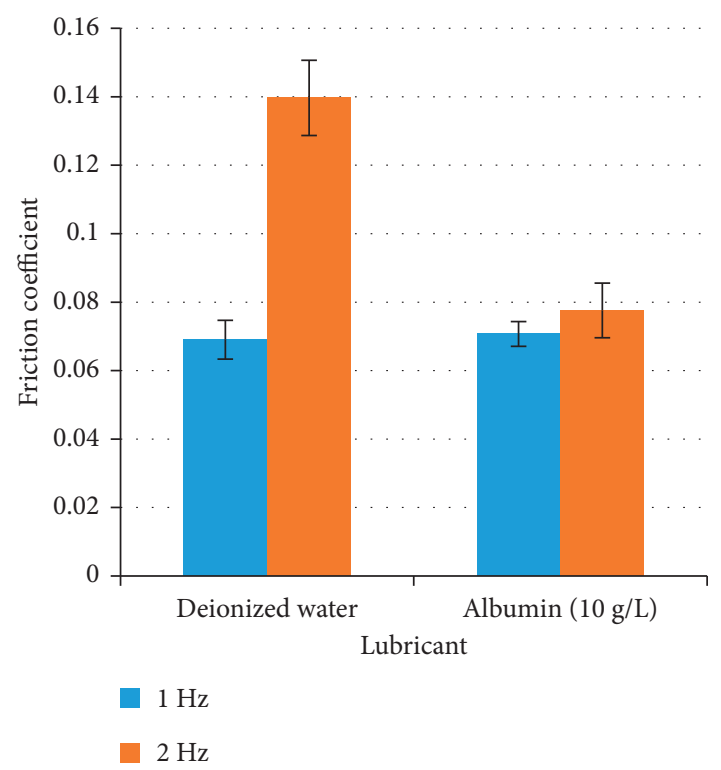

Figure 5: Effect of speed under $1 \mathrm{~N}$ load condition.

2.4. Measurement of Mechanical Properties. Figure 2 shows unconfined compression tests to evaluate the samples. The hydrogel samples were cut with a circular knife, and then cylinder-shaped samples for mechanical measurements were obtained. The diameter of the cylindrical samples was $5 \mathrm{~mm}$. The samples were compressed between two stainless steel plates at $0.6 \mathrm{~mm} / \mathrm{min}$. The compression modulus of the PVA hydrogel sample was measured using

$$
E^{\prime}=\frac{\sigma}{\varepsilon}
$$

where $E^{\prime}$ is the compression modulus, $\sigma$ is the stress, and $\varepsilon$ is the strain.

The UMT-3 Micro-Tribometer (CETR Inc., USA) was also used in the mechanical measurements. It has a force 


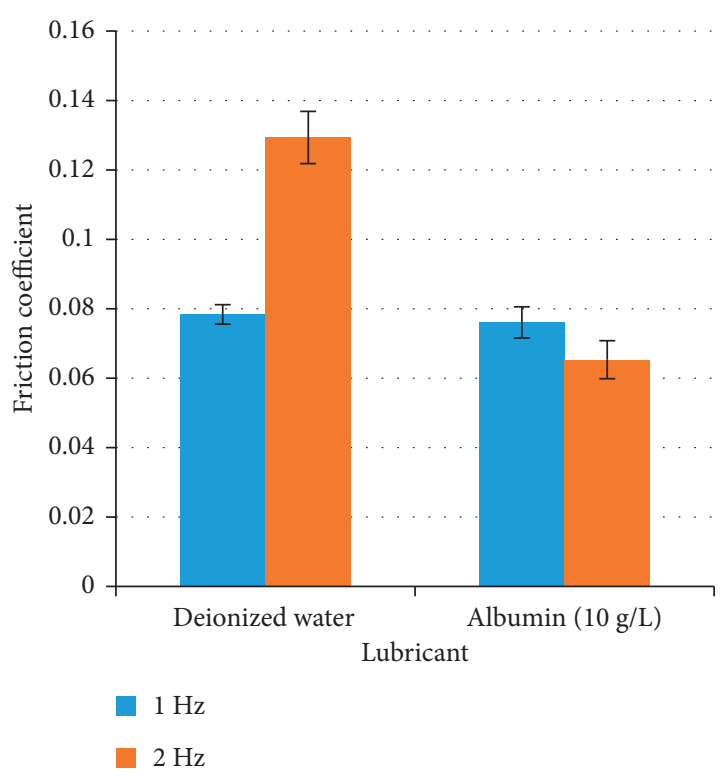

FIgURE 6: Effect of speed under $2 \mathrm{~N}$ load condition.

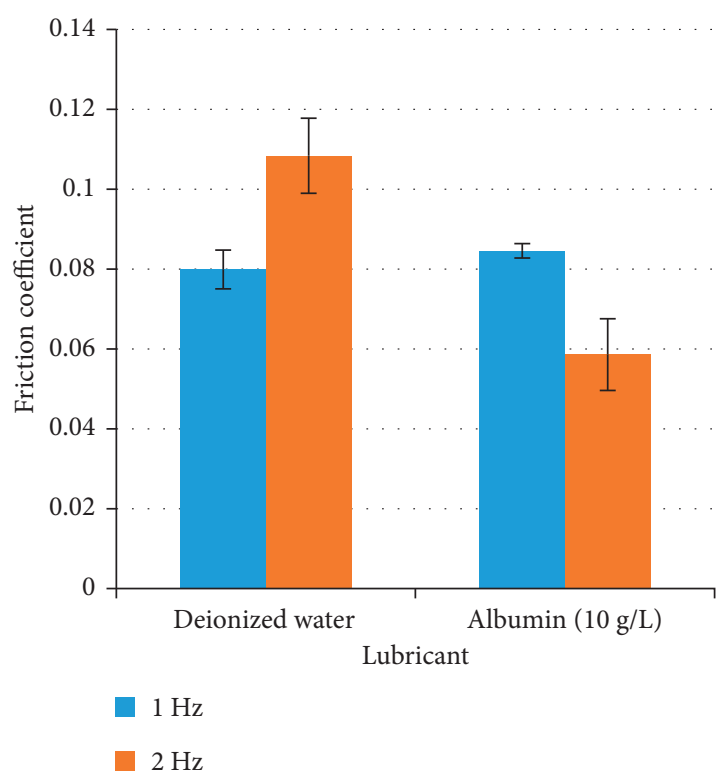

Figure 7: Effect of speed under $3 \mathrm{~N}$ load condition.

resolution of $0.1 \mathrm{mN}$ and a speed resolution of $0.001 \mathrm{~mm} / \mathrm{s}$. It could fulfill the requirements of these tests. The displacement in the $z$-axis was also measured in the reciprocating frictional test.

\section{Results}

3.1. Effects of Load. Figures 3 and 4 show friction changes for samples lubricated with deionized water or albumin under three loading levels. The coefficient of fraction data (Figure 3) indicates that the coefficient increased at $1 \mathrm{~Hz}$ and decreased when the speed was higher. The test was conducted at the lowest loading level $(1 \mathrm{~N})$, and the highest coefficient of friction was 0.14 at $1 \mathrm{~Hz}$.

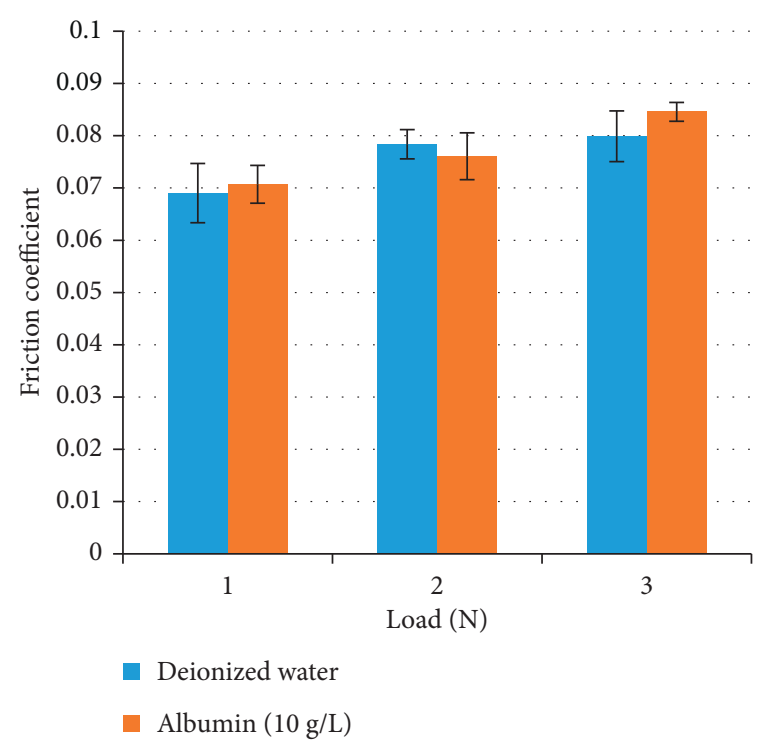

FIgURE 8: Effect of lubrication condition at a speed of $1 \mathrm{~Hz}$.

When the samples were tested in albumin solution, the state of friction shifted abruptly in disparate trends at different speeds. This result reflected the diverse trend of friction coefficient with the lowest value of 0.58 at the three loading levels. This indicates that when the albumin solution was used as the lubricant, the influence of loading on the coefficient of friction reversed.

3.2. Effects of Speed. The bar graph shows the effects of speed on the coefficient of friction when the PVA hydrogel was tested with the stainless steel ball. Figures 5-7 indicate that the coefficient of friction increased as the speed increased from $1 \mathrm{~Hz}$ to $2 \mathrm{~Hz}$ under the lubrication of deionized water. This situation is unchanged across the three loading levels. Albumin and water had similar trends. The coefficient of friction increased and was slightly altered while the load was in the lowest level of $1 \mathrm{~N}$. As the load increased from $1 \mathrm{~N}$ to $2 \mathrm{~N}$, the coefficient of friction markedly dropped from 0.076 to 0.065 . When the load was $3 \mathrm{~N}$ and the speed increased, the friction condition shifted dramatically from high to low (fallen by one-third). Figure 6 shows a similar phenomenon when the load was $2 \mathrm{~N}$ with albumin. The results indicated that the speed had an opposing effect on the friction with different lubricants at high loading levels.

3.3. Effects of Lubrication. The bar charts of Figures 8 and 9 show the impact of lubricant on the tribological properties of the PVA hydrogel as an artificial cartilage. The bar graph in Figure 8 shows a similar growth trend of the friction state lubricated by deionized water as that with albumin. The absolute value of the coefficient of friction did not indicate significant differences between these two lubricants. As the reciprocating motion frequency increased from $1 \mathrm{~Hz}$ to $2 \mathrm{~Hz}$, a divergent view was seen (Figure 9). The coefficient of friction tested in deionized water had different values. They were twice as high as those tested in albumin. This result means that the maximum value of friction coefficient was reduced by lubrication of the albumin solution. 


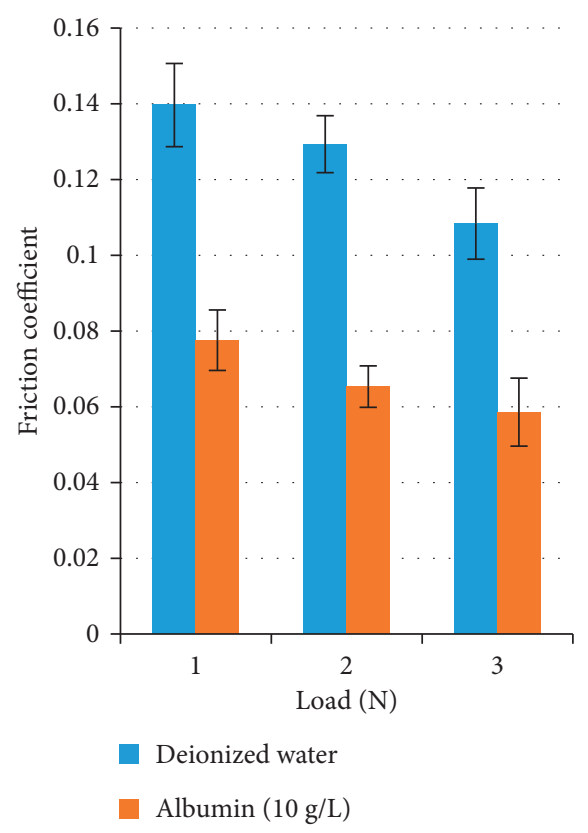

FIgURE 9: Effect of lubrication condition at a speed of $2 \mathrm{~Hz}$.

3.4. Surface Morphology. A long-term test was conducted to examine the wear properties of the sample in albumin. According to the scanning electron micrograph of the sample (Figure 10), the surface had wear with two obvious wear tracks. The porous surface of PVA hydrogel samples changed.

3.5. Mechanical Properties. Figure 11 shows the relationship between stress and strain as the PVA hydrogel samples were compressed. The contact force was measured as strain moved from $50 \%$ to $70 \%$. The changes in compression modulus are also shown in Figure 11. When the strain was $60 \%$ and the stress was $0.68 \mathrm{MPa}$, the compression modulus was $1.14 \pm 0.15 \mathrm{MPa}$.

3.6. The Relationship between Frictional and Mechanical Properties. The displacement of the $z$-axis could be measured during each reciprocating frictional test. The data were processed by data viewer 2.16 of UMT-3 Micro-Tribometer (CETR Inc., USA). Figures 12 and 13 show that the coefficient of friction and $z$-axis displacement changed during the 6-minute test $(2 \mathrm{~Hz}, 3 \mathrm{~N}$, and albumin solution). The value variation of $z$-axis displacement implies deformation at the surface of the sample. At the beginning, a load of $3 \mathrm{~N}$ was applied to the surface. It started to deform slightly. On the contrary, the coefficient of starting friction rose sharply and reached a peak value of 0.16 . The sample surface then deformed and reached a peak value of 48.31 at 32 seconds.

3.7. Surface Roughness. After the frictional tests were completed, the surface morphology of the sample surface was examined to reveal the broken surface. The surface roughness was tested before and after these tests under albumin solution (AS) and deionized water (DW). Figure 14

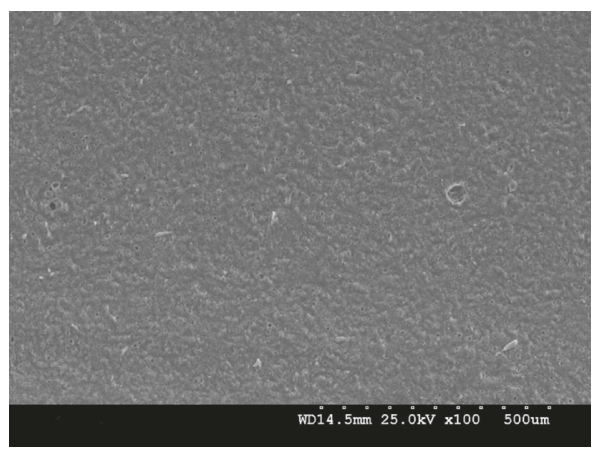

(a)

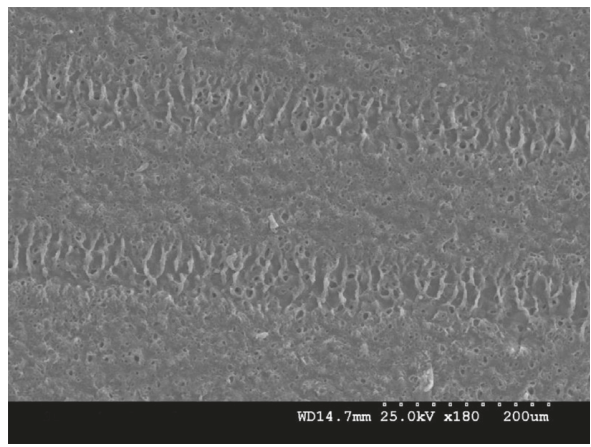

(b)

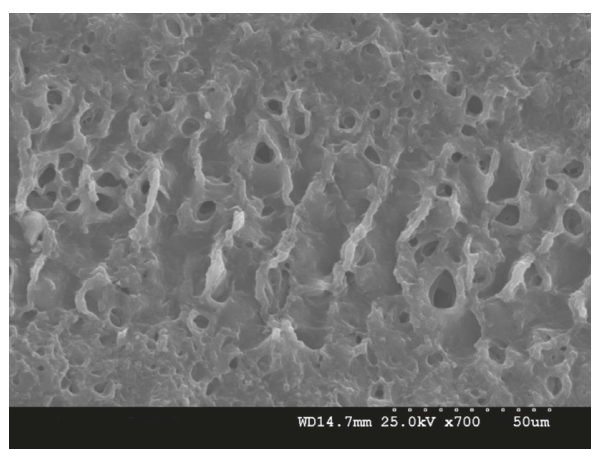

(c)

FIGURE 10: Surface morphology examination by scanning electron microscopy. (a) Unworn surface of PVA hydrogel. (b) Scanning electron micrograph of the wear surface, two wear tracks had formed. (c) The enlarged view of a wear track.

shows that the surface roughness changed slightly in all tests. The albumin solution could reduce the surface change for its better lubricating properties.

\section{Discussion}

Substantial attention has been given to the potential influence of diverse loading levels on the friction condition of natural and artificial cartilages $[27,28]$. In some studies, the coefficient of friction declines with increasing load [22]. Some people speculated that this is related to the testing conditions or testing configurations on which the samples were measured [29]. The decline in the friction coefficient reached a turning point if the testing condition of these experiments could be changed. Due to the measurement 


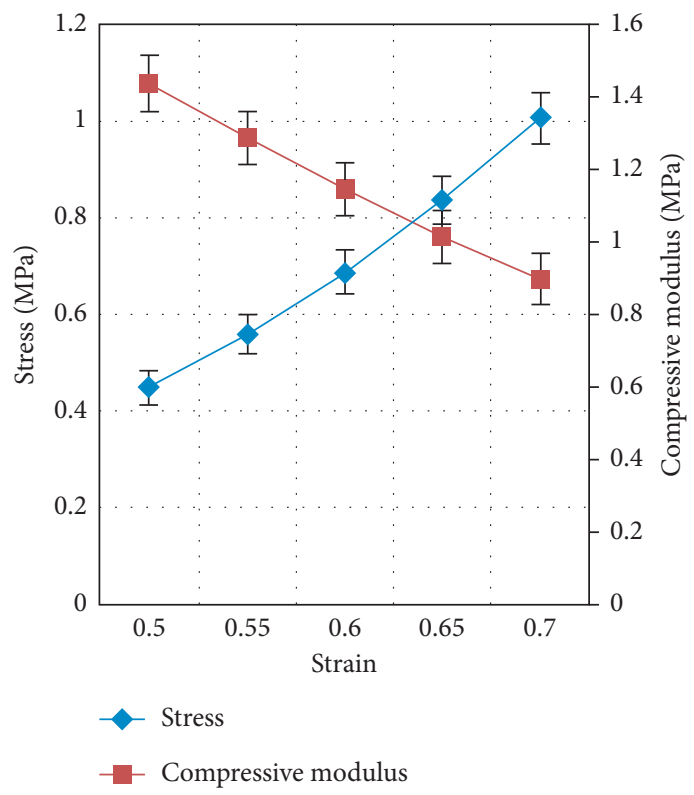

FIGURE 11: Stress-strain relationship and compressive modulus.

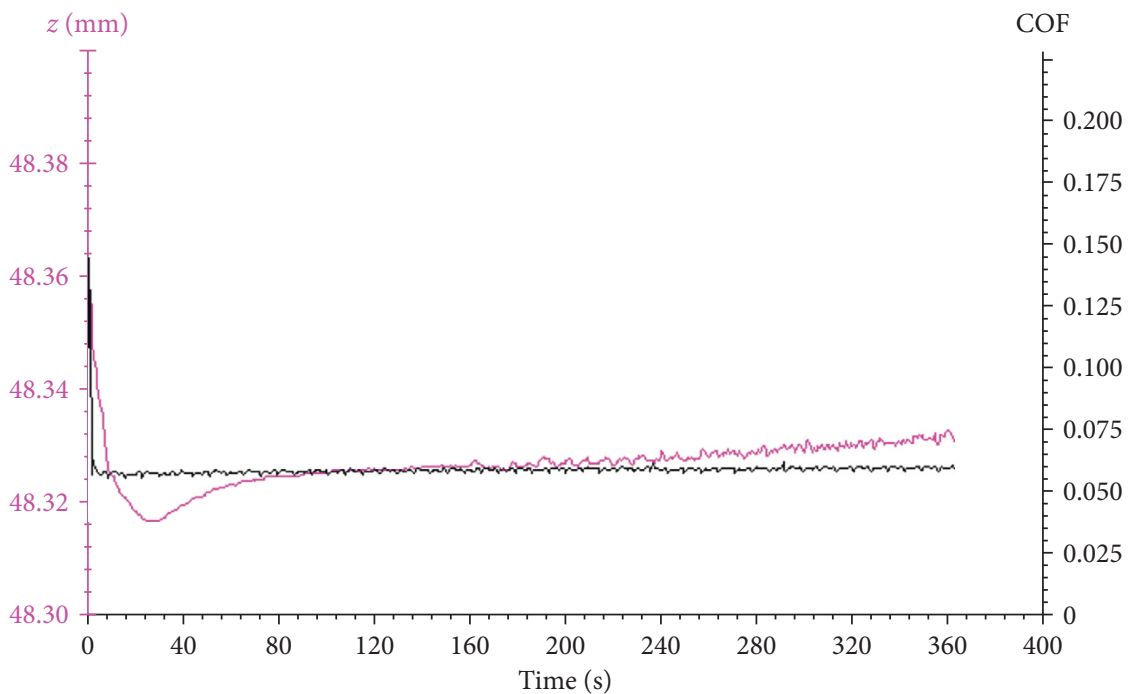

FiguRe 12: The change of friction coefficient and $z$-axis displacement $(2 \mathrm{~Hz}, 3 \mathrm{~N}$, and albumin solution).

range of the force transducer, three loads were used. The increasing load gradually decreased the coefficient of friction at high speed. However, the increasing load also led to an increase in the coefficient of friction while the speed was low. When the speed was low, the PVA hydrogel easily deformed because the load could be applied longer than at high speed in the same sample surface. The high deformation would also result in a large contact area in the frictional pair, and the coefficient of friction would increase concurrently.

Another important parameter was the turning point mentioned above. The turning point could change the prevailing trend of the friction, and it might be related to the reciprocating speed. Moreover, the phenomenon of hydration and dehydration of the articular cartilage had significant effects on the declining trend of friction [30]. In this study, there was no single trend of the coefficient of friction with the stainless steel ball against the artificial cartilage. When deionized water was used as the lubricant and the load was increased, the variation in friction showed the opposite trend as albumin: the coefficient increased at $1 \mathrm{~Hz}$ and reduced at $2 \mathrm{~Hz}$.

In the conditions with different stages of osteoarthritis (OA), the damaged state of natural cartilage of human and the use of hyaluronic acid and dipalmitoyl phosphatidylcholine affect the start-up friction in the initial sample movement [31]. When a lubricant solution of hyaluronic acid was used, the coefficient of friction indicated an obvious decline compared to Ringer's solution. Forsey et al. found a friction reduction as high as 0.146 when the lubricant was 


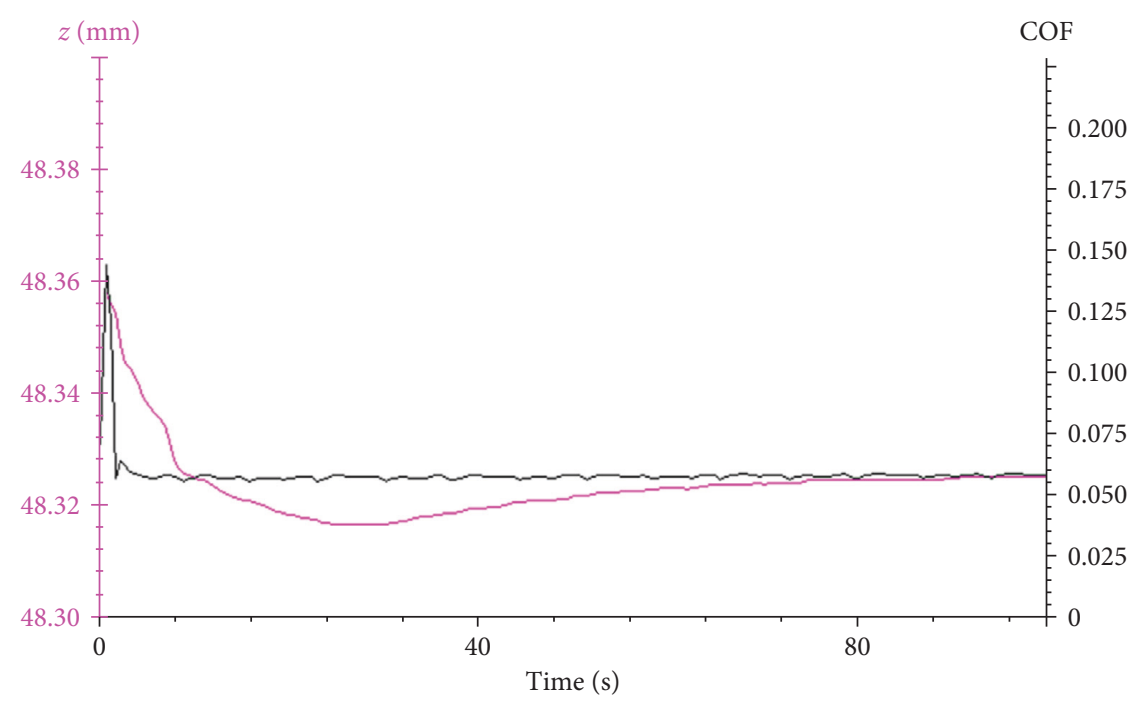

Figure 13: Enlarged view of the first 100 seconds ( $2 \mathrm{~Hz}, 3 \mathrm{~N}$, and albumin solution).

changed from Ringer's to hyaluronic acid solution. Hyaluronic acid is a part of the synovial fluid in the natural joint. Dipalmitoyl phosphatidylcholine is a good boundary lubricant on natural cartilage surfaces [32]. Here, if the speed was high, there was also a reduction in the coefficient of friction, while the lubricant of deionized water was replaced with albumin solution. However, this trend was dissimilar to that tested under deionized water. Human joints move at a variety of speeds. The results indicated that albumin in synovial fluid might not reduce friction when people move slowly. These other constituents of synovial fluid should be examined by more sophisticated methods to determine how speed, load, and lubrication can reduce joint friction.

Hills [33] showed that the benefits of hyaluronic acid as an effective lubricant are attributed to its wetting properties. It can attract a large amount of water to the synovial fluid. Our data showed that albumin is a regular protein with no wetting properties in the lubricating liquid. Albumin is easily dissolved in water or saline. The nonwetting action of albumin means that albumin could be used as a boundary lubricant instead of changing the surface wettability, which is related to the surface energy. Previous research [31] has shown that hyaluronic acid and lipids are a better lubricant for the joint. Albumin could be used in such situations to reduce the friction and wear while clarifying its main function.

In the hydrodynamic area, the role of albumin and bovine serum in lubrication has been studied in film thickness measurements conducted on a ball-on-disc device with a cobalt-chromium metal femoral head [25]. They found that the aggregation and absorbance of the protein occur at the surface with a thin protein film to reduce the friction. The friction pair would have low solid contact between their surfaces with resulting low friction. This is because the film thicknesses are higher at low speeds than at high speeds. It is interesting to compare their results with our results. Figure 3 shows that the coefficient of friction is lower at $1 \mathrm{~Hz}$ when the albumin solution was used as the lubricant and the load was $1 \mathrm{~N}$. However, when the load increased to $2 \mathrm{~N}$ or $3 \mathrm{~N}$, the coefficient of friction

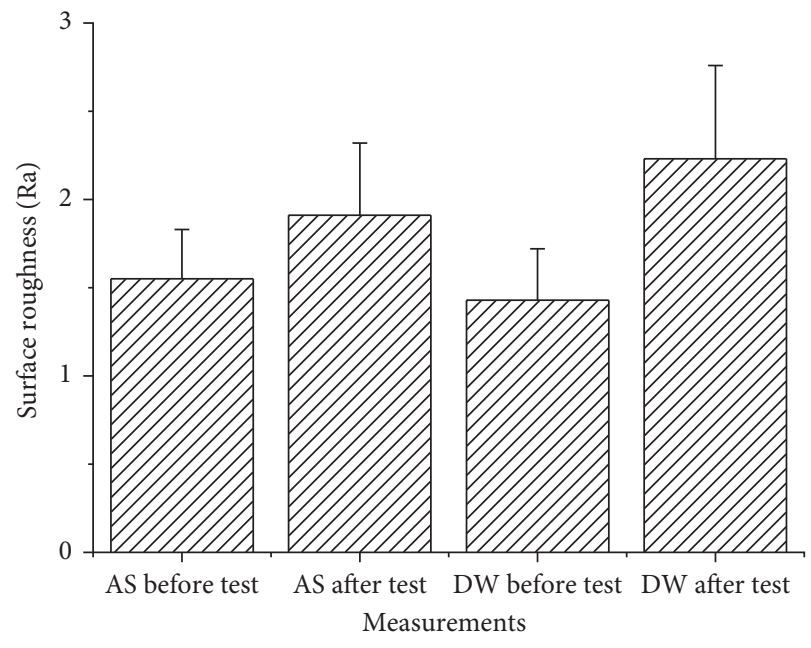

FIGURE 14: Surface roughness of the samples.

was no longer low at $1 \mathrm{~Hz}$. These results can partially be explained by the film thickness changes mentioned previously [25]. In addition, the load factor should be considered in future studies of film thickness measurements.

The evaluation of the worn PVA hydrogel sample showed that the top layer of the sample failed. The porous structure of the surface changed. The scanning electron microscopy results revealed that the main wear mechanisms were surface fatigue wear. As a result, the fluid support that can greatly reduce this friction would decrease during reciprocating motion [34].

In this study, the variation in the $z$-axis displacement indicated that the surface deformation can evaluate the relationship between surface depression and frictional properties. The results of friction coefficient and the $z$-axis displacement showed that the surface deformation changed but lagged behind the transformation of the friction coefficient. When the coefficient of friction reached a peak value of 0.16 , the surface sample began to deform. When the 
coefficient of friction changed steadily, the deformation of the sample had its largest value $(48.31 \mathrm{~mm}$ at 32 seconds).

In the previous study, the PVA hydrogel is regarded as a kind of porous material with many water components that can be used in biomedical areas [35]. While the load was applied, the water in its 3D space was pushed out and deformation increased. As a result, friction conditions got worse. The interstitial fluid pressurization of the biphasic lubrication mechanism in natural articular cartilage has been tested $[36,37]$ and can also be applied to this artificial cartilage material. If the water phase of the cartilage takes most proportion of the load, then the solid phase of the cartilage can be protected with lower pressure and the friction coefficient would be reduced.

Here, the relationship between the surface deformation and the friction condition was unclear. When the test duration was more than 32 seconds, a very interesting phenomenon was seen: the sample's surface had recovered, and the friction coefficient has no distinct change in Figure 12. The data show that the coefficient of friction increased gradually from 0.056 at 32 seconds to 0.06 at 6 minutes, but the sample's recovery from deformation occurred steadily over the same period. There may be other important factors that greatly affect the frictional properties of the sample other than biphasic lubrication.

According to the biphasic lubrication mechanism, the friction coefficient of the samples decreases if the surface is recovered with the water phase. However, in our research, when the deformation decreased 32 seconds later, the friction coefficient increased smoothly from 0.056 to 0.060 .

This indicates that the surface damage might have a greater influence than the water phase support induced from surface deformation. The destruction of the hydrogel surface might explain the increasing coefficient of friction during the testing period. Whether the surface change or the surface deformation or other factors dominate this observation requires further study.

\section{Conclusions}

The effects of surface mechanical deformation and bovine serum albumin on the tribological properties of the PVA hydrogel as artificial cartilage were studied. The results showed that the lubrication of albumin had a crucial effect on the tribological properties of artificial cartilage. The influence of the load on coefficient of friction was reversed in albumin. When the speed was high $(2 \mathrm{~Hz})$, the friction reduced, and the changing trend of the friction coefficient was the same as that lubricated by deionized water. Surface wear appeared, and the sample surface had tracks after wear testing. The mechanical and frictional results showed that even though the surface deformation could recover in the water phase, the porous structure returned, and the coefficient of friction continued to increase simultaneously. The biphasic lubrication effects may not be the only factor dominating the excellent friction properties of the PVA hydrogel; the surface change, or other factors, might also have more important effects.

\section{Conflicts of Interest}

The authors declare that they have no conflicts of interest.

\section{Acknowledgments}

This research was supported by the National Natural Science Foundation of China (no. 51605238), Shandong Province Key Research and Development Plan (2017GGX30108), Qingdao Original Innovation Program basic research for application project (17-1-1-92-jch), and the University Science and Technology Project of Shandong Province (J16LB07).

\section{References}

[1] F. Liu, J. Fisher, and Z. Jin, "Effect of motion inputs on the wear prediction of artificial hip joints," Tribology International, vol. 63, pp. 105-114, 2013.

[2] H. C. Amstutz, P. Grigoris, M. R. Safran, M. J. Grecula, P. A. Campbell, and T. P. Schmalzried, "Precision-fit surface hemiarthroplasty for femoral head osteonecrosis. Long-term results," Journal of Bone and Joint Surgery, British Volume, vol. 76, no. 3, pp. 423-427, 1994.

[3] N. R. Lestrange, "Bipolar arthroplasty for 496 hip fractures," Clinical Orthopaedics and Related Research, no. 251, pp. 7-19, 1990.

[4] R. Leyshon and J. Matthews, "Acetabular erosion and the Monk "hard top" hip prosthesis," Journal of Bone and Joint Surgery, British Volume, vol. 66, no. 2, pp. 172-174, 1984.

[5] J. D'Arcy and M. Devas, "Treatment of fractures of the femoral neck by replacement with the Thompson prosthesis," Journal of Bone and Joint Surgery, British Volume, vol. 58, no. 3, pp. 279-286, 1976.

[6] V. C. Mow and W. M. Lai, "Recent developments in synovial joint biomechanics," SIAM Review, vol. 22, no. 3, pp. 275-317, 1980.

[7] Y. S. Pan, D. S. Xiong, and R. Y. Ma, "A study on the friction properties of poly(vinyl alcohol) hydrogel as articular cartilage against titanium alloy," Wear, vol. 262, no. 7-8, pp. 1021-1025, 2007.

[8] L. Bostan, A. M. Trunfio-Sfarghiu, L. Verestiuc et al., "Mechanical and tribological properties of poly(hydroxyethyl methacrylate) hydrogels as articular cartilage substitutes," Tribology International, vol. 46, no. 1, pp. 215-224, 2012.

[9] J. L. Holloway, K. L. Spiller, A. M. Lowman, and G. R. Palmese, "Analysis of the in vitro swelling behavior of poly(vinyl alcohol) hydrogels in osmotic pressure solution for soft tissue replacement," Acta Biomaterialia, vol. 7, no. 6, pp. 2477-2482, 2011.

[10] M. M. Blum and T. C. Ovaert, "Experimental and numerical tribological studies of a boundary lubricant functionalized poro-viscoelastic PVA hydrogel in normal contact and sliding," Journal of the Mechanical Behavior of Biomedical Materials, vol. 14, pp. 248-258, 2012.

[11] M. I. Baker, S. P. Walsh, Z. Schwartz, and B. D. Boyan, "A review of polyvinyl alcohol and its uses in cartilage and orthopedic applications," Journal of Biomedical Materials Research Part B: Applied Biomaterials, vol. 100, no. 5, pp. 1451-1457, 2012.

[12] K. W. Ng, P. A. Torzilli, R. F. Warren, and S. A. Maher, "Characterization of a macroporous polyvinyl alcohol scaffold for the repair of focal articular cartilage defects," Journal of 
Tissue Engineering and Regenerative Medicine, vol. 8, no. 2, pp. 164-168, 2014.

[13] J. A. Stammen, S. Williams, D. N. Ku, and R. E. Guldberg, "Mechanical properties of a novel PVA hydrogel in shear and unconfined compression," Biomaterials, vol. 22, no. 8, pp. 799-806, 2001.

[14] D. Baykal, J. S. Day, D. J. Jaekel, J. Katta, K. Mansmann, and S. M. Kurtz, "Tribological evaluation of hydrogel articulations for joint arthroplasty applications," Journal of the Mechanical Behavior of Biomedical Materials, vol. 14, pp. 39-47, 2012.

[15] V. M. Sardinha, L. L. Lima, W. D. Belangero, C. A. Zavaglia, V. P. Bavaresco, and J. R. Gomes, "Tribological characterization of polyvinyl alcohol hydrogel as substitute of articular cartilage," Wear, vol. 301, no. 1-2, pp. 218-225, 2013.

[16] M. A. Accardi, D. Dini, and P. M. Cann, "Experimental and numerical investigation of the behaviour of articular cartilage under shear loading-interstitial fluid pressurisation and lubrication mechanisms," Tribology International, vol. 44, no. 5 , pp. 565-578, 2011.

[17] M. Kobayashi, Y.-S. Chang, and M. Oka, "A two year in vivo study of polyvinyl alcohol-hydrogel (PVA-H) artificial meniscus," Biomaterials, vol. 26, no. 16, pp. 3243-3248, 2005.

[18] S. H. Hyon, W. I. Cha, and Y. Ikada, "Preparation of transparent poly(vinyl alcohol) hydrogel," Polymer Bulletin, vol. 22, no. 2, pp. 119-122, 1989.

[19] A. M. Patel and M. Spector, "Tribological evaluation of oxidized zirconium using an articular cartilage counterface: a novel material for potential use in hemiarthroplasty," Biomaterials, vol. 18, no. 5, pp. 441-447, 1997.

[20] R. A. Brand, "Joint contact stress: a reasonable surrogate for biological processes?," Iowa Orthopaedic Journal, vol. 25, p. 82,2005 .

[21] A. Unsworth, "Recent developments in the tribology of artificial joints," Tribology International, vol. 28, no. 7 , pp. 485-495, 1995.

[22] J. Katta, S. S. Pawaskar, Z. M. Jin, E. Ingham, and J. Fisher, "Effect of load variation on the friction properties of articular cartilage," Proceedings of the Institution of Mechanical Engineers, Part J: Journal of Engineering Tribology, vol. 221, no. 3, pp. 175-181, 2007.

[23] R. J. Covert, R. D. Ott, and D. N. Ku, "Friction characteristics of a potential articular cartilage biomaterial," Wear, vol. 255, no. 7-12, pp. 1064-1068, 2003.

[24] C. W. Myant and P. Cann, "The effect of transient conditions on synovial fluid protein aggregation lubrication," Journal of the Mechanical Behavior of Biomedical Materials, vol. 34, pp. 349-357, 2014.

[25] J. Fan, C. Myant, R. Underwood, P. Cann, and A. Hart, "Inlet protein aggregation: a new mechanism for lubricating film formation with model synovial fluids," Proceedings of the Institution of Mechanical Engineers, Part H: Journal of Engineering in Medicine, vol. 225, no. 7, pp. 696-709, 2011.

[26] T. Hatakeyema, J. Uno, C. Yamada, A. Kishi, and H. Hatakeyama, "Gel-sol transition of poly(vinyl alcohol) hydrogels formed by freezing and thawing," Thermochimica Acta, vol. 431, no. 1-2, pp. 144-148, 2005.

[27] G. A. Ateshian, M. A. Soltz, R. L. Mauck, I. M. Basalo, C. T. Hung, and W. M. Lai, "The role of osmotic pressure and tension-compression nonlinearity in the frictional response of articular cartilage," Transport in Porous Media, vol. 50, no. 1-2, pp. 5-33, 2003.

[28] L. L. Malcom, An Experimental Investigation of the Frictional and Deformational Responses of Articular Cartilage Interfaces to Static and Dynamic Loading, University of California, San Diego, CA, USA, 1976.

[29] J. Katta, Z. Jin, E. Ingham, and J. Fisher, "Biotribology of articular cartilage-a review of the recent advances," Medical Engineering \& Physics, vol. 30, no. 10, pp. 1349-1363, 2008.

[30] J. Katta, Z. Jin, E. Ingham, and J. Fisher, "Effect of nominal stress on the long term friction, deformation and wear of native and glycosaminoglycan deficient articular cartilage," Osteoarthritis and Cartilage, vol. 17, no. 5, pp. 662-668, 2009.

[31] R. W. Forsey, J. Fisher, J. Thompson, M. H. Stone, C. Bell, and E. Ingham, "The effect of hyaluronic acid and phospholipid based lubricants on friction within a human cartilage damage model," Biomaterials, vol. 27, no. 26, pp. 4581-4590, 2006.

[32] B. A. Hills and B. D. Butler, "Surfactants identified in synovial fluid and their ability to act as boundary lubricants," Annals of the Rheumatic Diseases, vol. 43, no. 4, pp. 641-648, 1984.

[33] B. A. Hills, "Oligolamellar lubrication of joints by surface active phospholipid," Journal of Rheumatology, vol. 16, no. 1, pp. 82-91, 1989.

[34] G. A. Ateshian, H. Wang, and W. M. Lai, "The role of interstitial fluid pressurization and surface porosities on the boundary friction of articular cartilage," Journal of Tribology, vol. 120, no. 2, pp. 241-248, 1998.

[35] S. Azmi, S. I. A. Razak, M. R. Abdul Kadir et al., "Reinforcement of poly(vinyl alcohol) hydrogel with halloysite nanotubes as potential biomedical materials," Soft Materials, vol. 15, no. 1, pp. 45-54, 2017.

[36] S. R. Oungoulian, K. M. Durney, B. K. Jones, C. S. Ahmad, C. T. Hung, and G. A. Ateshian, "Wear and damage of articular cartilage with friction against orthopedic implant materials," Journal of Biomechanics, vol. 48, no. 10, pp. 1957-1964, 2015

[37] M. Caligaris and G. A. Ateshian, "Effects of sustained interstitial fluid pressurization under migrating contact area, and boundary lubrication by synovial fluid, on cartilage friction," Osteoarthritis and Cartilage, vol. 16, no. 10, pp. 1220-1227, 2008. 

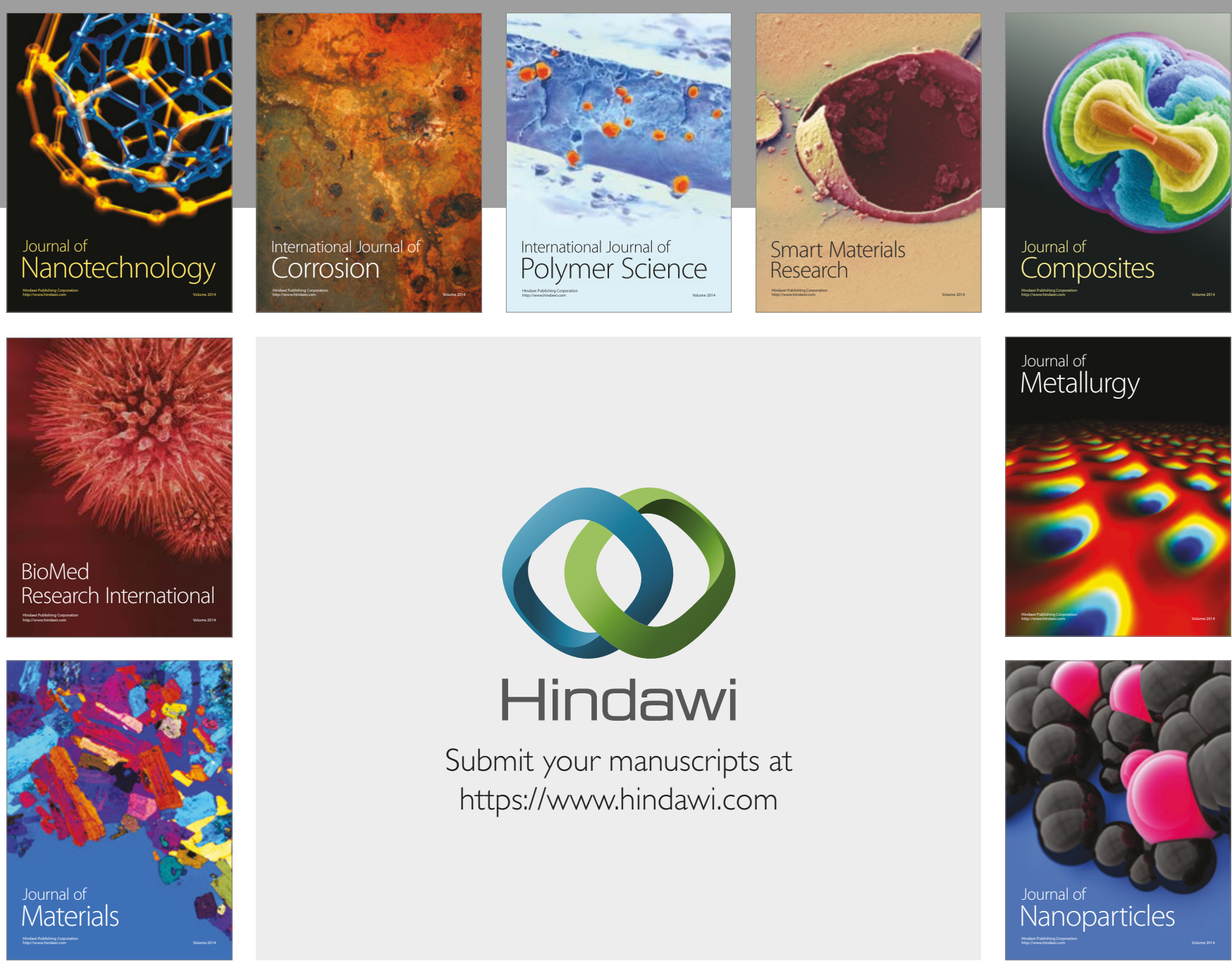

\section{Hindawi}

Submit your manuscripts at

https://www.hindawi.com
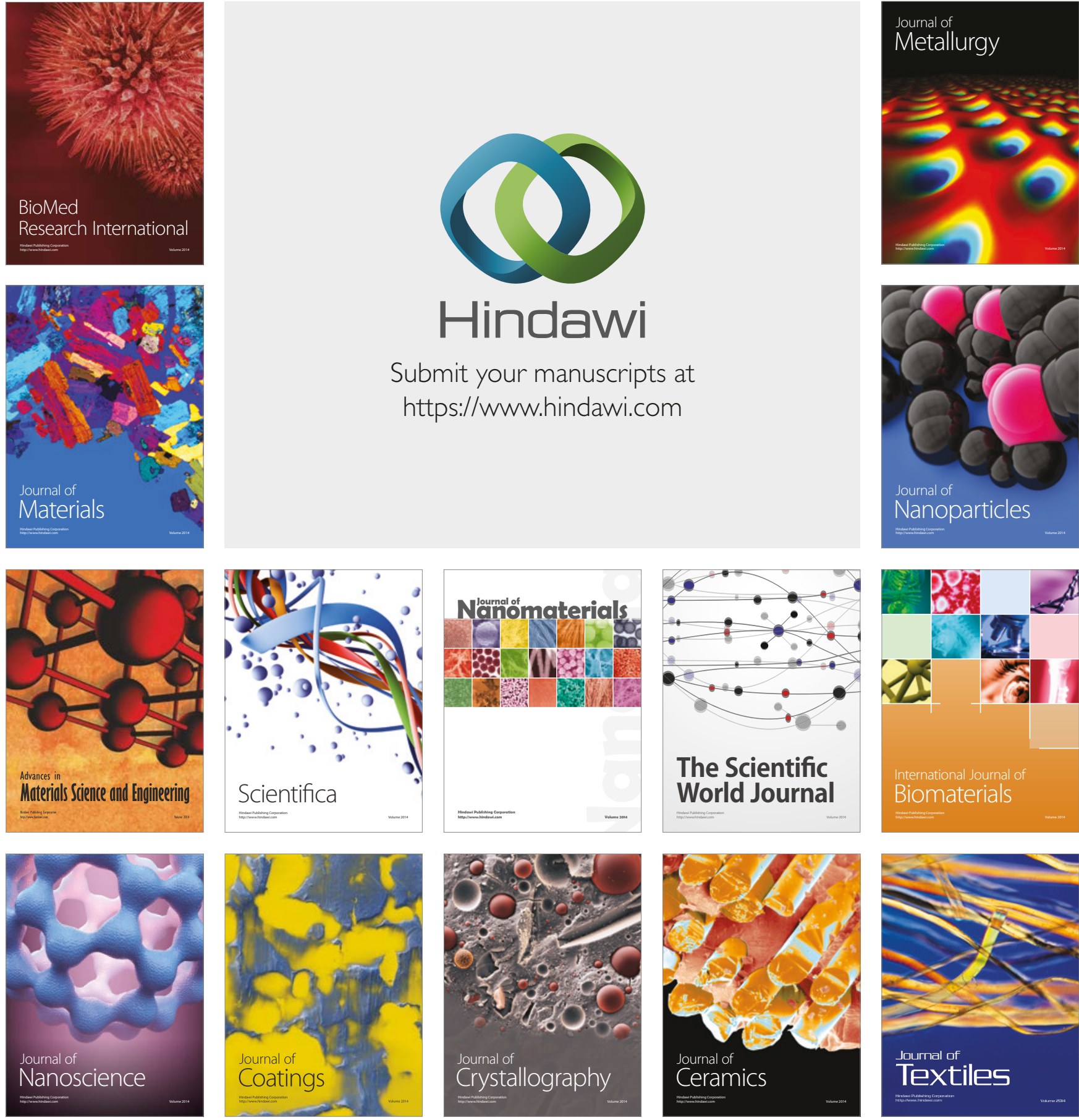

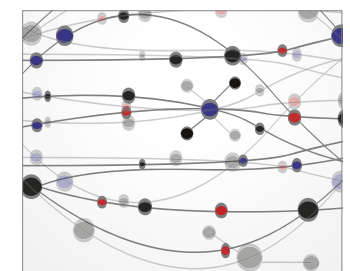

The Scientific World Journal
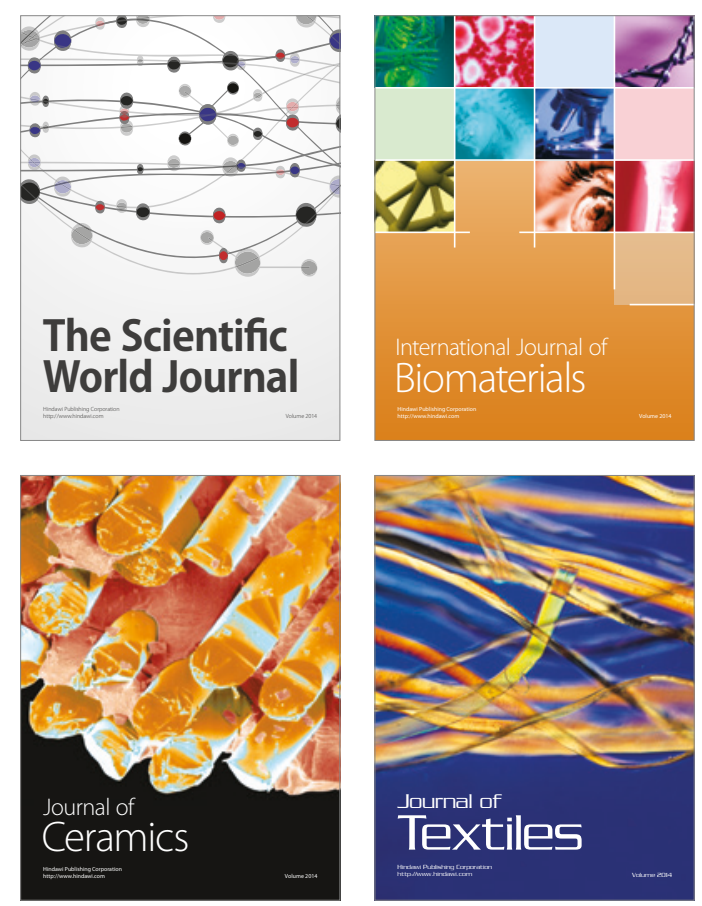